\title{
Testing the nature of the black hole candidate in GRO J1655-40 with the relativistic precession model
}

\author{
Cosimo Bambi ${ }^{\mathrm{a}}$ \\ Department of Physics, Center for Field Theory and Particle Physics, Fudan University, Shanghai 200433, China
}

Received: 12 September 2014 / Accepted: 8 April 2015 / Published online: 24 April 2015

(C) The Author(s) 2015. This article is published with open access at Springerlink.com

\begin{abstract}
Quasi-periodic oscillations (QPOs) are a common feature in the X-ray flux of stellar-mass black hole candidates, but their exact origin is not yet known. Recently, some authors have pointed out that data of GRO J1655-40 simultaneously show three QPOs that nicely fit in the relativistic precession model. However, they find an estimate of the spin parameter that disagrees with the measurement of the disk's thermal spectrum. In the present work, I explore the possibility of using the relativistic precession model to test the nature of the black hole candidate in GRO J1655-40. If properly understood, QPOs may become a quite powerful tool to probe the spacetime geometry around black hole candidates, especially if used in combination with other techniques. It turns out that the measurements of the relativistic precession model and of the disk's thermal spectrum may be consistent if we admit that the black hole candidate in GRO J1655-40 is not of the Kerr type.
\end{abstract}

\section{Introduction}

In four-dimensional general relativity, uncharged black holes (BHs) are described by the Kerr solution and are completely characterized by only two parameters: the mass $M$ and the spin angular momentum $J$. This is the result of the wellknown "no-hair" theorem [1-3]. $M$ and $J$ cannot be completely arbitrary, but they must satisfy the condition for the existence of the event horizon $|a| \leq M$, where $a=J / M$ is the spin parameter. Astrophysical BHs, if they exist, should be well described by the Kerr metric: initial deviations from the Kerr geometry are expected to be quickly radiated away through the emission of gravitational waves [4,5], an initially non-vanishing electric charge would be shortly neutralized in their highly ionized environment [6], while the presence of the accretion disk is completely negligible in most cases.

\footnotetext{
a e-mail: bambi@fudan.edu.cn
}

Astronomical observations have discovered at least two classes of BH candidates: stellar-mass objects in X-ray binary systems with a mass $M \approx 5-20 M_{\odot}$, and super-massive bodies in galactic nuclei with a mass $M \sim 10^{5}-10^{9} M_{\odot}$ [7]. All these objects are thought to be the Kerr BHs of general relativity, but their actual nature is still to be verified. Robust measurements of the masses of these objects can be obtained from dynamical methods, by studying the orbital motion of gas or of individual stars around them. Such measurements are the main argument to support the Kerr BH hypothesis, because these objects are so heavy that they cannot be explained otherwise without introducing new physics. The non-observation of electromagnetic radiation emitted by the possible surface of these objects may also be interpreted as an indication for the existence of an event horizon [8,9] (but see [10,11]). However, there is no evidence that the spacetime geometry around them is described by the Kerr solution.

The nature of astrophysical BH candidates may be potentially tested with the already available X-ray data, because the features of the electromagnetic radiation emitted by the gas of the accretion disk can provide information on the spacetime geometry around these compact objects (for a review, see e.g. $[12,13])$. The study of the disk's thermal spectrum (continuum-fitting method) [14-16] and the analysis of the profile of the broad $\mathrm{K} \alpha$ iron line [17-19] are today the only two relatively mature techniques to probe the metric around $\mathrm{BH}$ candidates. They have been developed to infer the spin parameter of these objects under the assumption of the Kerr spacetime, but more recently they have been extended to check the Kerr background [20-28]. The main problem to test the Kerr $\mathrm{BH}$ paradigm with these techniques is that it is extremely difficult to get independent estimates of the spin parameter and of possible deviations from the Kerr solution. In other words, one can usually only constrain a combination of the spin and of possible deviations, because the properties of the radiation emitted by the gas in the accretion disk around a non-Kerr object with a certain spin can be very similar to the ones produced in the spacetime of a Kerr BH with 
different spin. The possibility of combining the continuumfitting method and the iron line analysis for the same object has been discussed in Ref. [29]. For some BH solutions, the combination of the two approaches is not very helpful and the Kerr metric cannot be unambiguously tested. In other $\mathrm{BH}$ backgrounds, the study of the disk's thermal spectrum and the analysis of the iron line profile of a specific source can do the job, but quite accurate measurements are usually necessary. The possibility of using the estimate of the power of transient or steady jets with the measurements from the continuum-fitting method has been explored in Ref. [30,31]. While the approach seems to be promising, the mechanisms responsible for the formation of these jets are not known and different interpretations lead to different conclusions. In the future, high resolution sub-mm observations will be hopefully able to detect the "shadow" of nearby super-massive $\mathrm{BH}$ candidates, opening a new window to test the spacetime geometry around these objects [32-35].

Quasi-periodic oscillations (QPOs) are a very promising tool to get precise information on the spacetime geometry around stellar-mass $\mathrm{BH}$ candidates. They are seen as peaks in the X-ray power density spectra of the source. At present, however, the exact physical mechanism responsible for the production of these QPOs is not understood and several different scenarios have been proposed, including relativistic precession models [36-38], diskoseismology models [3941], resonance models [42-44], and $p$-mode oscillations of an accretion torus $[45,46]$. In most scenarios, the frequencies of the QPOs are directly related to the characteristic orbital frequencies of a test particle, which are determined only by the background metric and are independent of the complicated astrophysical processes of the accretion. While such a correlation with the fundamental frequencies of the spacetime may sound quite artificial at first, it is possible to show that there is indeed a direct relation between these frequencies and the ones of the oscillation modes of the fluid accretion flow. The significant advantage of the use of QPOs with respect to other techniques is that the frequencies of the QPOs can be measured with high accuracy, and therefore they can potentially be used to get very precise measurements of the parameters of the spacetime geometry of the compact object. Attempts to use the QPOs to test the Kerr metric around $\mathrm{BH}$ candidates are reported in [47-49]. However, since we do not know the exact mechanism responsible for these oscillations, such a powerful approach cannot yet be used. Different models relate the fundamental frequencies of the background and the observed frequencies of the QPOs in a different way, and current X-ray data are not able to select the correct model and rule out the others.

Very recently, some authors have pointed out that the X-ray data of GRO J1655-40 nicely fit in the relativistic precession model [50]. The key-point is that this source is the only one for which three simultaneous QPOs have been observed. In the Kerr spacetime, the three fundamental frequencies of the background metric (orbital frequency, radial epicyclic frequency, and vertical epicyclic frequency) depend on the radius $r$, the $\mathrm{BH}$ mass $M$, and the $\mathrm{BH}$ spin parameter $a$. Assuming that the three observed QPOs are generated at the same radius $r$, one has a system of three equations with three unknown variables $(r, M$, and $a)$. The system of the equations can therefore be solved to find $r, M$, and $a$, which can be determined with a quite small uncertainty due to the high precision of the measurement of the frequencies. The authors of Ref. [50] find that the inferred value of $M$ is in agreement with the value obtained by dynamical methods in Ref. [51]. In support of the relativistic precession model, the authors of Ref. [50] show also that the X-ray data of GRO J1655-40 with two simultaneous QPOs can be nicely interpreted as two of the three frequencies generated at radii $r$ larger than the one found in the data with three frequencies. However, their spin measurement is not consistent with the one obtained from the continuum-fitting method in Ref. [52].

The aim of the present paper is to investigate the possibility of using the data and the interpretation of Ref. [50] to test the spacetime geometry around the $\mathrm{BH}$ candidate in GRO J1655-40. For this purpose, it is convenient to consider a metric more general than the Kerr one, with one (or more) deformation parameter(s). The latter is used to measure possible deviations from the Kerr background, which must be recovered when the deformation parameter vanishes. Now one needs an independent measurement of the mass of the $\mathrm{BH}$ candidate, so that it is possible to solve the system of equations of the three frequencies to find the three unknown quantities ( $r, a$, and the deformation parameter). The result is an allowed region on the spin-deformation parameter plane, just like the authors of Ref. [50] find an allowed region on the mass-spin plane. The strong correlation between the spin and possible deviations from the Kerr solution found with other approaches is present even here, but the size of the allowed region is much smaller, supporting the idea that, if properly understood, QPOs can be a very powerful tool to probe the spacetime geometry around $\mathrm{BH}$ candidates. In order to check the validity of this result, the latter is compared with the allowed region on the spin-deformation parameter plane inferred from the study of the disk's thermal spectrum of GRO J1655-40 [52]. It turns out that the disagreement between the two measurements found in the Kerr metric cannot be solved if we believe in the mass measurement of Ref. [51]. However, a different measurement of the mass of the BH candidate in GRO J1655-40 is reported in [52]. If we believe in the mass measurement of this work, the one found in the Kerr background with the relativistic precession model in [50] is wrong, while it is possible to reconcile the QPO measurement of the spin with the disk's thermal spectrum analysis if we allow for deviations from the Kerr geometry. 
In the latter case, the non-vanishing deformation parameter would be compatible with the one inferred in the second paper in $[30,31]$ from the combination of the measurements of the disk spectrum and the estimates of the power of steady jets. While that may be accidental, if the relativistic precession model turns out to be right we may suspect that the continuum-fitting method regularly overestimates the spin parameter or even speculate on the violation of the Kerr BH paradigm.

The content of the paper is as follows. In Sect. 2, I briefly review the relativistic precession model and the results of Ref. [50], valid in the Kerr background. In Sect. 3, I apply this approach to the rotating Bardeen BH metric [53-55] and to the Johannsen-Psaltis background [56] to find an allowed region on the spin-deformation parameter plane. In Sect. 4, I discuss these results, which are also compared with the constraints that can be obtained from the continuum-fitting method. Summary and conclusions are reported in Sect. 5 . Throughout the paper, I use units in which $G_{\mathrm{N}}=c=1$, unless stated otherwise.

\section{The relativistic precession model}

The relativistic precession model was originally proposed to explain QPOs in low-mass X-ray binaries with a neutron star and was then extended to systems with stellarmass BH candidates [36-38]. It does not really explain the origin of the QPOs, but it simply relates the observed frequencies of the QPOs with the three fundamental frequencies of the background metric. The latter are the Keplerian frequency of equatorial circular orbits (orbital frequency $v_{\phi}$ ) and the frequencies of small perturbations along the radial and vertical direction around the equatorial circular orbit (respectively, the radial epicyclic frequency $v_{r}$ and the vertical epicyclic frequency $v_{\theta}$ ). In the Kerr metric, these frequencies can be written in analytic form and are given by

$v_{\phi}=\left(\frac{1}{2 \pi}\right) \frac{M^{1 / 2}}{r^{3 / 2} \pm a M^{1 / 2}}$,

$v_{r}=v_{\phi}\left(1-\frac{6 M}{r} \pm \frac{8 a M^{1 / 2}}{r^{3 / 2}}-\frac{3 a^{2}}{r^{2}}\right)$,

$v_{\theta}=v_{\phi}\left(1 \mp \frac{4 a M^{1 / 2}}{r^{3 / 2}}+\frac{3 a^{2}}{r^{2}}\right)$,

where the upper (lower) sign is for the case of corotating (counterrotating) orbits. From these three frequencies, one can find the periastron precession frequency $v_{\mathrm{p}}$ and the nodal precession frequency $v_{\mathrm{n}}$, given by

$$
v_{\mathrm{p}}=v_{\phi}-v_{r}
$$$$
v_{\mathrm{n}}=v_{\phi}-v_{\theta} \text {. }
$$

All these frequencies depend on three parameters; that is, the radius of the orbit $r$ and the two parameters of the background geometry, the $\mathrm{BH}$ mass $M$, and the $\mathrm{BH}$ spin parameter $a$.

In X-ray binaries with a $\mathrm{BH}$ candidate, observations have detected low-frequency QPOs of different nature (type-A, type-B, and type-C) in the range $\sim 0.1-30 \mathrm{~Hz}$, and highfrequency QPOs at $\sim 100-400 \mathrm{~Hz}$. The latter may be seen in pairs and they are therefore called lower and upper highfrequency QPOs. The crucial point is to find the correct relation between the fundamental frequencies of the background metric and the ones of the observed QPOs. In Ref. [50], the authors propose the following interpretation (which is not exactly the original proposal of the relativistic precession model in [36-38]). The low-frequency type-C QPO $v_{\mathrm{C}}$ would correspond to the nodal precession frequency $v_{\mathrm{n}}$, while the lower high-frequency QPO $\nu_{\mathrm{L}}$ and the upper high-frequency $\mathrm{QPO} \nu_{\mathrm{U}}$ would be associated, respectively, to the periastron precession frequency $v_{\mathrm{p}}$ and to the orbital frequency $v_{\phi}$ :

$v_{\mathrm{C}}=v_{\mathrm{n}}, \quad v_{\mathrm{L}}=v_{\mathrm{p}}, \quad v_{\mathrm{U}}=v_{\phi}$.

The case of the BH candidate in GRO J1655-40 is special, because it is the only BH system for which we have data with three simultaneous QPOs. The low-frequency type-C QPO used in [50] was identified in Ref. [57], while the two high-frequency QPOs were found in [58]. Since one sees simultaneously the three frequencies, it is possible to argue that they may be associated to oscillations of the fluid flow at the same radial coordinate. In this way, one can solve the system of equations of the three frequencies $\left(v_{\mathrm{C}}, \nu_{\mathrm{L}}\right.$, and $\left.v_{\mathrm{U}}\right)$ to find the three unknown variables $(r, M$, and $a$ ). The system of equations cannot be solved analytically and therefore one has to find the three parameters numerically. Here I use a different approach with respect to Ref. [50] and I compute the $\chi$-square as follows:

$$
\begin{aligned}
\chi_{0}^{2}(r, M, a)= & \frac{\left(v_{\mathrm{C}}-v_{\mathrm{n}}\right)^{2}}{\sigma_{\mathrm{C}}^{2}}+\frac{\left(v_{\mathrm{L}}-v_{\mathrm{p}}\right)^{2}}{\sigma_{\mathrm{L}}^{2}} \\
& +\frac{\left(v_{\mathrm{U}}-v_{\phi}\right)^{2}}{\sigma_{\mathrm{U}}^{2}} .
\end{aligned}
$$

For GRO J1655-40, we have [50]

$$
\begin{array}{cl}
v_{\mathrm{C}}=17.3 \mathrm{~Hz}, & \sigma_{\mathrm{C}}=0.1 \mathrm{~Hz}, \\
\nu_{\mathrm{L}}=298 \mathrm{~Hz}, & \sigma_{\mathrm{L}}=4 \mathrm{~Hz}, \\
v_{\mathrm{U}}=441 \mathrm{~Hz}, & \sigma_{\mathrm{U}}=2 \mathrm{~Hz} .
\end{array}
$$

The minimum of $\chi_{0}^{2}$ (which should be zero in this case, as the system of equations has always a solution) gives the estimate of $r, M$, and $a$, while the intervals defined by $\chi_{0}^{2}=\chi_{0, \min }^{2}+\Delta \chi_{0}^{2}$ give the ranges of $r, M$, and $a$ at the confidence level (C.L.) set by $\Delta \chi_{0}^{2}$. In the case of three degrees of freedom, $\Delta \chi_{0}^{2}=3.53,8.03$, and 14.16 correspond, respectively, to $68.3,95.4$, and $99.7 \%$ C.L., which are the proba- 


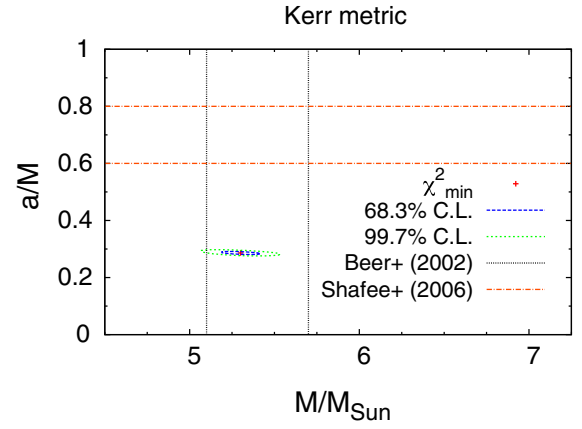

Fig. 1 Estimate of the mass $M$ and of the spin parameter $a / M$ of the BH candidate in GRO J1655-40 with the relativistic precession model of Ref. [50] and under the assumption of the Kerr background. With the approach discussed in Sect. 2, the result is $M / M_{\odot}=5.30 \pm 0.11$ and $a / M=0.286 \pm 0.006$ (68.3\% C.L.). The vertical black thin-

bility intervals designated as 1,2, and 3 standard deviation limits.

Following this procedure, one finds the plots in Fig. 1. The final result for the mass and the spin parameter is $M / M_{\odot}=$ $5.30 \pm 0.11$ and $a / M=0.286 \pm 0.006$ (68.3\% C.L.). The estimate of the mass is consistent with the value inferred by optical observations in Ref. [51], $M / M_{\odot}=5.4 \pm 0.3$, which corresponds to the black thin-dotted lines in the left panel in Fig. 1. However, in the literature there is also another mass measurement of the BH candidate in GRO J1655-40, $M / M_{\odot}=6.3 \pm 0.3$, reported in [52]. The orange dasheddotted curve corresponds instead to the measurement of the spin parameter inferred via the continuum-fitting method in Ref. [52], $a / M=0.7 \pm 0.1 .^{1}$ Such a measurement does depend on the BH mass $M$, but in Fig. 1 I show only the best estimate for $a / M$ assuming that the mass (which is an input parameter in the continuum-fitting method) obtained by optical measurements is correct. In Ref. [52], the authors use $M / M_{\odot}=6.3 \pm 0.3$, not the one of Ref. [51], but the effect on the estimate of the spin is not large and cannot solve the disagreement between the relativistic precession model and the continuum-fitting method. The measurement of the frequencies of QPOs can potentially provide very precise estimates of the mass $M$ and the spin parameter $a$ compared to other techniques. However, the measurements from the relativistic precession model and the continuum-fitting method provide inconsistent results, which means that either one of the two approaches provides an erroneous value of the spin parameter $a / M$, or both. In the next sections, I will check if the two techniques can give consistent results if we allow for deviations from the Kerr background.

\footnotetext{
1 Actually, the measurement in Ref. [52] is $a / M=0.70 \pm 0.05$ at 1 sigma, but since it was one of the first measurements obtained with the continuum-fitting method by the CfA group, in later studies using this result the uncertainty has been conservatively doubled by the same authors.
}

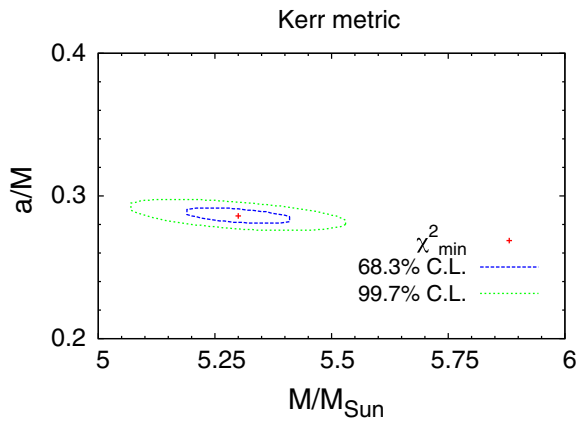

dotted lines are the boundaries of the optical measurement of the mass of this object found in Ref. [51], while the orange dashed-dotted curve is the boundary of the allowed region for the spin parameter via the continuum-fitting method obtained in Ref. [52]. The right panel is just the enlargement of the left one. See the text for more details

\section{Testing the Kerr nature of GRO J1655-40}

A common approach to test the nature of astrophysical $\mathrm{BH}$ candidates and constrain possible deviations from the Kerr solution is to consider a more general background, which includes the Kerr metric as a special case. In addition to the mass $M$ and the spin parameter $a$, the spacetime geometry is characterized by at least one more parameter, which is used to measure possible deviations from the Kerr background. The idea is to infer $M, a$, and such a deformation parameter from observational data and check if the latter require a vanishing deformation parameter; that is, the compact object is a Kerr BH. On the contrary, if it turns out that observations require a non-vanishing deformation parameter, the $\mathrm{BH}$ candidate may not be a Kerr BH.

Let us now revise the relativistic precession model in a generic stationary, axisymmetric, and asymptotically flat spacetime. The line element of the spacetime can be written in the canonical form,

$\mathrm{d} s^{2}=g_{t t} \mathrm{~d} t^{2}+g_{r r} \mathrm{~d} r^{2}+g_{\theta \theta} \mathrm{d} \theta^{2}+2 g_{t \phi} \mathrm{d} t \mathrm{~d} \phi+g_{\phi \phi} \mathrm{d} \phi^{2}$,

where the metric components are independent of the $t$ and $\phi$ coordinates, which implies the existence of two constants of motion: the conserved specific energy at infinity, $E$, and the conserved $z$-component of the specific angular momentum at infinity, $L_{z}$. This fact allows one to write the $t$ - and $\phi$ component of the 4-velocity of a test particle as

$\dot{t}=\frac{E g_{\phi \phi}+L_{z} g_{t \phi}}{g_{t \phi}^{2}-g_{t t} g_{\phi \phi}}, \quad \dot{\phi}=-\frac{E g_{t \phi}+L_{z} g_{t t}}{g_{t \phi}^{2}-g_{t t} g_{\phi \phi}}$.

From the conservation of the rest mass, $g_{\mu \nu} \dot{x}^{\mu} \dot{x}^{\nu}=-1$, we can write

$g_{r r} \dot{r}^{2}+g_{\theta \theta} \dot{\theta}^{2}=V_{\mathrm{eff}}\left(r, \theta, E, L_{z}\right)$, 
where the effective potential $V_{\text {eff }}$ is given by

$V_{\text {eff }}=\frac{E^{2} g_{\phi \phi}+2 E L_{z} g_{t \phi}+L_{z}^{2} g_{t t}}{g_{t \phi}^{2}-g_{t t} g_{\phi \phi}}-1$.

Circular orbits on the equatorial plane are located at the zeros and the turning points of the effective potential: $\dot{r}=\dot{\theta}=0$, which implies $V_{\text {eff }}=0$, and $\ddot{r}=\ddot{\theta}=0$, requiring, respectively, $\partial_{r} V_{\text {eff }}=0$ and $\partial_{\theta} V_{\text {eff }}=0$. From these conditions, one can obtain the orbital angular velocity $\Omega_{\phi}=\mathrm{d} \phi / \mathrm{d} t, E$, and $L_{z}$ of the test particle:

$$
\begin{aligned}
\Omega_{\phi} & =\frac{-\partial_{r} g_{t \phi} \pm \sqrt{\left(\partial_{r} g_{t \phi}\right)^{2}-\left(\partial_{r} g_{t t}\right)\left(\partial_{r} g_{\phi \phi}\right)}}{\partial_{r} g_{\phi \phi}} \\
E & =-\frac{g_{t t}+g_{t \phi} \Omega_{\phi}}{\sqrt{-g_{t t}-2 g_{t \phi} \Omega_{\phi}-g_{\phi \phi} \Omega_{\phi}^{2}}}, \\
L_{z} & =\frac{g_{t \phi}+g_{\phi \phi} \Omega_{\phi}}{\sqrt{-g_{t t}-2 g_{t \phi} \Omega_{\phi}-g_{\phi \phi} \Omega_{\phi}^{2}}},
\end{aligned}
$$

where in $\Omega_{\phi}$ the sign is $+(-$ ) for corotating (counterrotating) orbits. The orbital frequency is simply $v_{\phi}=\Omega_{\phi} / 2 \pi$. The orbits are stable under small perturbations if $\partial_{r}^{2} V_{\text {eff }} \leq 0$ and $\partial_{\theta}^{2} V_{\text {eff }} \leq 0$.

The radial and vertical epicyclic frequencies can be quickly computed by considering small perturbations around circular equatorial orbits, respectively, along the radial and vertical direction. If $\delta_{r}$ and $\delta_{\theta}$ are the small displacements around the mean orbit (i.e. $r=r_{0}+\delta_{r}$ and $\theta=\pi / 2+\delta_{\theta}$ ), we find they are governed by the following differential equations:

$$
\begin{gathered}
\frac{\mathrm{d}^{2} \delta_{r}}{\mathrm{~d} t^{2}}+\Omega_{r}^{2} \delta_{r}=0, \\
\frac{\mathrm{d}^{2} \delta_{\theta}}{\mathrm{d} t^{2}}+\Omega_{\theta}^{2} \delta_{\theta}=0,
\end{gathered}
$$

where

$$
\begin{aligned}
& \Omega_{r}^{2}=-\frac{1}{2 g_{r r} \dot{t}^{2}} \frac{\partial^{2} V_{\mathrm{eff}}}{\partial r^{2}}, \\
& \Omega_{\theta}^{2}=-\frac{1}{2 g_{\theta \theta} \dot{t}^{2}} \frac{\partial^{2} V_{\mathrm{eff}}}{\partial \theta^{2}} .
\end{aligned}
$$

The radial epicyclic frequency is thus $v_{r}=\Omega_{r} / 2 \pi$ and the vertical one is $\nu_{\theta}=\Omega_{\theta} / 2 \pi$.

As a first example of non-Kerr background, we can consider the Bardeen BH metric [53-55]. In Boyer-Lindquist coordinates, the non-vanishing metric coefficients are

$g_{t t}=-\left(1-\frac{2 m r}{\Sigma}\right), \quad g_{t \phi}=-\frac{2 a m r \sin ^{2} \theta}{\Sigma}$,

$g_{\phi \phi}=\left(r^{2}+a^{2}+\frac{2 a^{2} m r \sin ^{2} \theta}{\Sigma}\right) \sin ^{2} \theta$,

$g_{r r}=\frac{\Sigma}{\Delta}, \quad g_{\theta \theta}=\Sigma$, where

$\Sigma=r^{2}+a^{2} \cos ^{2} \theta, \quad \Delta=r^{2}-2 m r+a^{2}$,

$m=M\left(\frac{r^{2}}{r^{2}+g^{2}}\right)^{3 / 2}$.

$g$ can be interpreted as the magnetic charge of a non-linear electromagnetic field or just as a quantity introducing a deviation from the Kerr metric. The position of the even horizon is given by the larger root of $\Delta=0$ and therefore there is a bound on the maximum value of the spin parameter, above which there are no BHs. The maximum value of $a$ is $M$ for $g / M=0$ (Kerr case), and decreases as $g / M$ increases. The black thin-dotted curve in the left panel of Fig. 2 is the boundary separating $\mathrm{BH}$ solutions (left bottom corner) and horizonless solutions (right top corner) on the plane $(a / M, g / M)$. Since the horizonless solutions are likely very unstable objects with a short lifetime due to the ergoregion instability, they can be safely ignored.

Now we have three equations for $v_{\mathrm{n}}, v_{\mathrm{p}}$, and $v_{\phi}$ and four unknown variables $(r, M, a$, and $g$ ). In order to solve the system, we need an independent estimate of the mass $M$. In this case, the $\chi$-square becomes

$\chi^{2}(r, a, g)=\min _{M}\left[\chi_{0}^{2}+\frac{\left(M-M_{\mathrm{opt}}\right)^{2}}{\sigma_{M}^{2}}\right]$,

where $\chi_{0}^{2}$ is given in Eq. (6) and the three degrees of freedom are now $r, a$, and $g$. For $M_{\mathrm{opt}}=5.4 M_{\odot}$ and $\sigma_{M}=0.3 M_{\odot}$ [51], the result is shown in the left panel of Fig. 2, where the constraints on the spin and on possible deviations from the Kerr solutions are

$a / M=0.279_{-0.036}^{+0.012}$,

$g / M<0.56$,

at the $68.3 \%$ C.L.

To check the genericity of this result found in the specific case of the Bardeen BH solution, it is convenient to repeat the same exercise with a different background metric. As second example, now I consider the Johannsen-Psaltis metric, whose non-vanishing metric coefficients in Boyer-Lindquist coordinates are [56]

$$
\begin{aligned}
g_{t t}= & -\left(1-\frac{2 M r}{\Sigma}\right)(1+h), \\
g_{t \phi}= & -\frac{4 a M r \sin ^{2} \theta}{\Sigma}(1+h), \\
g_{\phi \phi}= & \sin ^{2} \theta\left(r^{2}+a^{2}+\frac{2 a^{2} M r \sin ^{2} \theta}{\Sigma}\right) \\
& +\frac{a^{2}(\Sigma+2 M r) \sin ^{4} \theta}{\Sigma} h, \\
g_{r r}= & \frac{\Sigma(1+h)}{\Delta+a^{2} h \sin ^{2} \theta}, \quad g_{\theta \theta}=\Sigma,
\end{aligned}
$$




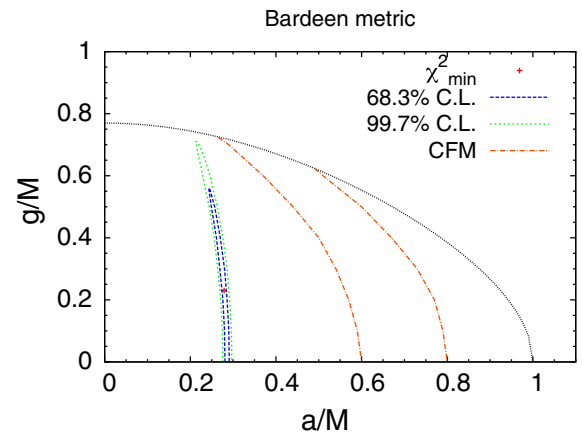

Fig. 2 Constraints on the spacetime geometry around the $\mathrm{BH}$ candidate in GRO J1655-40 with the relativistic precession model (blue dashed and green dotted lines) and the continuum-fitting method (orange dashed-dotted lines). The relativistic precession model assumes the

where

$\Sigma=r^{2}+a^{2} \cos ^{2} \theta, \quad \Delta=r^{2}-2 M r+a^{2}$,

$h=\sum_{k=0}^{\infty}\left(\epsilon_{2 k}+\frac{M r}{\Sigma} \epsilon_{2 k+1}\right)\left(\frac{M^{2}}{\Sigma}\right)^{k}$.

Such a metric has an infinite number of deformation parameters $\epsilon_{k}(k=0,1,2, \ldots)$. However, $\epsilon_{0}=\epsilon_{1}=0$ in order to recover the correct Newtonian limit, while $\epsilon_{2}$ is strongly constrained by Solar System experiments [56]. For the sake of simplicity, I will consider the case of a single deformation parameter $\epsilon_{3}$ and set to zero all the others. One can then define the counterpart of the $\chi$-square in Eq. (21):

$\chi^{2}\left(r, a, \epsilon_{3}\right)=\min _{M}\left[\chi_{0}^{2}+\frac{\left(M-M_{\mathrm{opt}}\right)^{2}}{\sigma_{M}^{2}}\right]$.

With the mass measurement of Ref. [51], the result is the plot in the right panel of Fig. 2. There is a quite pronounced correlation between the estimate of the spin and the deformation parameters, as shown by the thin but quite inclined position of the allowed region. The constraints are

$$
\begin{aligned}
a / M & =0.27_{-0.05}^{+0.06}, \\
\epsilon_{3} & =0.5_{-2.7}^{+2.1},
\end{aligned}
$$

at the $68.3 \%$ C.L.

\section{Discussion}

As shown in Fig. 2, while the relativistic precession interpretation of the data of GRO J1655-40 is perfectly consistent with the hypothesis that the spacetime around the BH candidate in this source is described by the Kerr metric, large deviations from the Kerr solutions are also allowed. One may wonder whether it is possible to solve the tension between the measurement inferred from this approach and the one obtained by the continuum-fitting method in Ref. [52]. The

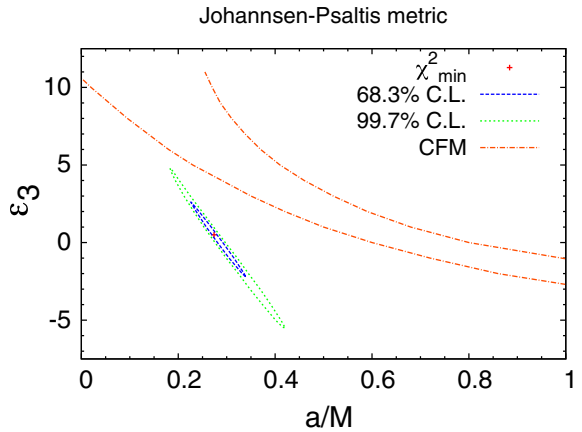

mass measurement $M / M_{\odot}=5.4 \pm 0.3$ reported in Ref. [51]. Left panel Bardeen background, where the black thin-dotted line is the boundary separating BHs from horizonless objects. Right panel Johannsen-Psaltis background with deformation parameter $\epsilon_{3}$. See the text for more details

estimate of the spin parameter found in the Kerr background in [52] can be quickly translated in an allowed region on the spin-deformation parameter plane by exploiting the fact that (at least for not too large deformation parameters) the disk's thermal spectrum around a deformed object with a certain spin is extremely similar to the one of a Kerr BH with different spin. Indeed, if we consider a non-Kerr BH metric and we fix the value of the deformation parameter, we can find a one-to-one correspondence between one of these objects and a Kerr BH whose disk's thermal spectrum is very similar.

With this spirit, if in the Kerr case the allowed spin parameter range is $0.6<a / M<0.8$, one can just find for any nonvanishing deformation parameter the spin of the non-Kerr BH with spectrum similar to a Kerr BH with $a / M=0.6$ and $a / M=0.8$. The result is the boundary of the allowed region in the spin-deformation parameter plane, which is the orange dashed-dotted line in Fig 2. Here the comparison of the spectra has been done using the $\chi$-square procedure of, for instance, Ref. [13]. The fact that there is not overlap between the allowed regions suggested by the relativistic precession approach and by the continuum-fitting method simply means that the tension between the two measurements cannot be solved assuming a different spacetime. One arrives at the same conclusions if the deformation parameter $\epsilon_{3}$ of the Johansenn-Psaltis background is replaced by higher order deformations. That has been explicitly checked for $\epsilon_{4}, \epsilon_{5}, \epsilon_{6}$, and $\epsilon_{7}$, and the general trend suggests it is correct for any $\epsilon_{k}$. While it is not possible to firmly exclude the possibility that some non-Kerr background can solve the tension between the two measurements, the failure of all these attempts suggests that such a possibility is at least not very natural.

For GRO J1655-40, in the literature there are also some estimates of its spin parameter with the $\mathrm{K} \alpha$ iron line method $[59,60]$. While the available data are not very good, these studies suggest that, in the case of a Kerr BH, the object would have a quite high value of the spin parameter, at the 

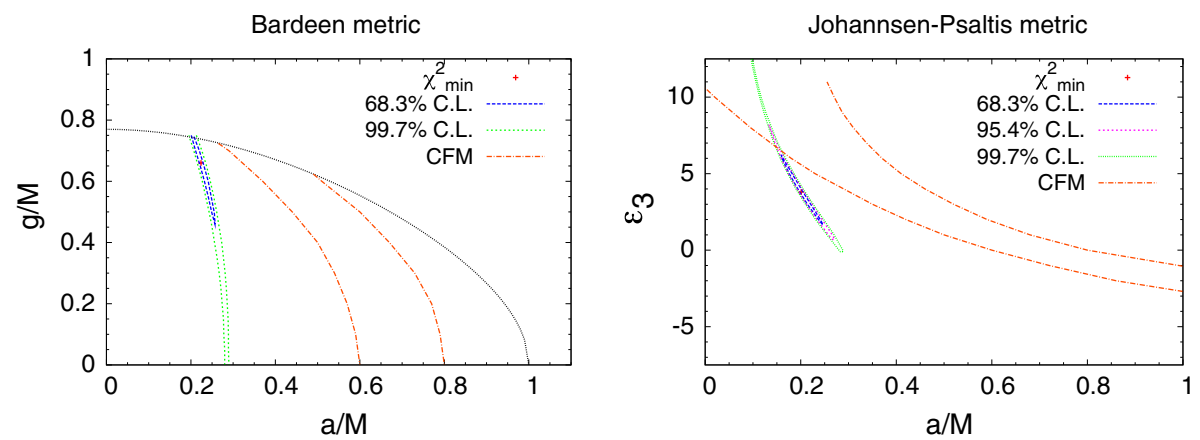

Fig. 3 As in Fig. 2, in the case in which the relativistic precession model uses the mass measurement $M / M_{\odot}=6.3 \pm 0.3$ reported in Ref. [52]

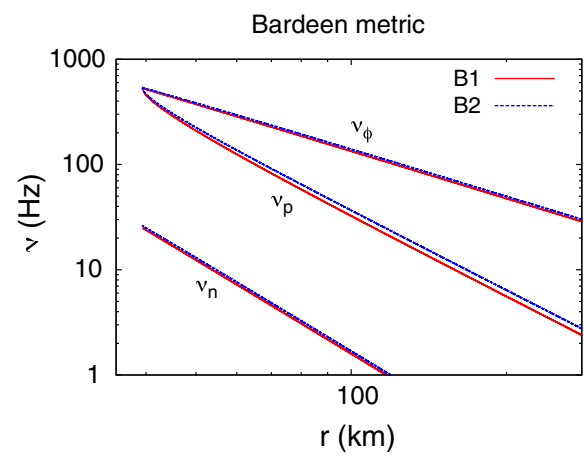

Fig. 4 Orbital frequency $v_{\phi}$, periastron precession frequency $v_{\mathrm{p}}$, and nodal precession frequency $v_{\mathrm{n}}$ as functions of the orbital radius $r$. Left panel Bardeen background with $M / M_{\odot}=5.40, a / M=0.279$, and $g / M=0.23(B 1)$ and with $M / M_{\odot}=5.95, a / M=0.243$,

level of $a / M \sim 0.9$ or even higher. It seems thus that the three approaches (relativistic precession model, disk's spectrum, iron line) give very different results. Following the study of Ref. [29], it is easy to conclude that for the Bardeen metric it is not possible to fix the tension between the three measurements, which continue to provide three different spins for any value of $g / M$. In the case of the Johannsen-Psaltis solution, the results from the continuum-fitting and the iron line analysis may be consistent in the case of a negative $\epsilon_{3}$ [29]. The compatibility between the relativistic precession interpretation and the other approaches seems, however, to be impossible.

Let us now consider what happens if we consider the mass measurement $M / M_{\odot}=6.3 \pm 0.3$ reported in [52], which is not consistent with the one of [51]. If we use this value as input parameter in the relativistic precession model, we find the plots in Fig. 3, respectively for the Bardeen (left panel) and Johannsen-Psaltis (right panel) backgrounds. In the Bardeen metric, the relativistic precession model and the disk's thermal spectrum are still inconsistent. In the Johannsen-Psaltis spacetime, we find an overlap between the 2-standard deviation region of the relativistic precession model and the 1-standard deviation limit of the continuumfitting method. The measurement of the relativistic precession model is

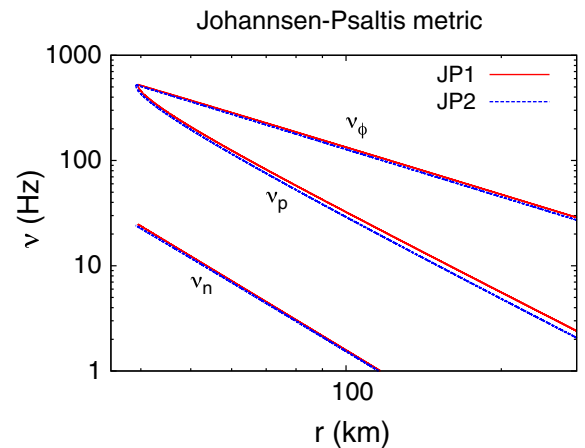

and $g / M=0.56$ (B2). Right panel Johannsen-Psaltis background with $M / M_{\odot}=5.42, a / M=0.274$, and $\epsilon_{3}=0.5(J P 1)$ and with $M / M_{\odot}=4.84, a / M=0.339$, and $\epsilon_{3}=-2.2(J P 2)$. See the text for more details

$$
\begin{aligned}
a / M & =0.20 \pm 0.04, \\
\epsilon_{3} & =3.8_{-2.1}^{+2.4},
\end{aligned}
$$

at $68 \%$ C.L. Such a measurement is consistent with the Kerr BH hypothesis within a 3-standard deviation limit (not within 1- and 2-standard deviation limits), but in combination with the analysis of the thermal spectrum of the disk favors a nonvanishing deformation parameter at the level of $\epsilon_{3} \sim 7$. It is worth noting that the same value was found in [31] from the combination of the measurements of the continuum-fitting method and of the power of steady jets for five $\mathrm{BH}$ candidates.

Lastly, it is important to stress that future X-ray satellites like LOFT can have the capabilities to test the relativistic precession model and hopefully provide robust and strong constraints on the nature of stellar-mass BH candidates. In particular, it would be extremely useful to have observations of QPOs at different radii. The three simultaneous QPOs in the available data seem to occur at a small radial coordinate, $r \sim 45 \mathrm{~km}$, which corresponds to a radius close to the innermost stable circular orbit of these spacetimes. Figure 4 shows the orbital frequency $v_{\phi}$, the periastron precession frequency $v_{\mathrm{p}}$, and the nodal frequency $v_{\mathrm{n}}$ as a function of the radial coordinate $r$ for the Bardeen and JohannsenPsaltis backgrounds, respectively left and right panels. In each panel, the red solid lines are the fundamental frequen- 
cies for the object at the point $\chi_{\min }^{2}$ in Fig. 2. $M / M_{\odot}=5.40$, $a / M=0.279$, and $g / M=0.23$ for the Bardeen case (B1) and $M / M_{\odot}=5.42, a / M=0.274$, and $\epsilon_{3}=0.5$ for the Johannsen-Psaltis metric (JP1). The blue dashed lines are instead the frequencies of an object on the $68.3 \%$ C.L. curve in Fig. 2. For the Bardeen solution (B2), the parameters are $M / M_{\odot}=5.95, a / M=0.243$, and $g / M=0.56$, and they belong to the object with maximum value of $g / M$ at $68.3 \%$ C.L. For the Johannsen-Psaltis metric (JP2), the parameters are $M / M_{\odot}=4.84, a / M=0.339$, and $\epsilon_{3}=-2.2$, and they are associated to the BH with a lowest possible value of $\epsilon_{3}$ allowed at $68.3 \%$ C.L. As shown in Fig. 4, the values of $v_{\phi}$ and $v_{\mathrm{n}}$ for different objects are similar even at larger radii, while the periastron precession frequency $v_{\mathrm{p}}$ seems to be more sensitive to the background metric. Very precise measurements of these frequencies at small and large radii may thus be a very powerful tool to distinguish Kerr BHs from other BH solutions.

\section{Summary and conclusions}

Astrophysical BH candidates are thought to be the Kerr BHs predicted in general relativity because they are so massive, compact, and dark that they cannot be explained otherwise without introducing new physics. Nevertheless, there are not yet observations capable of confirming this hypothesis. The properties of the electromagnetic radiation emitted by the gas in the inner part of the accretion disk can potentially provide information on the spacetime geometry around these compact objects and thus either confirm the predictions of general relativity or demand new physics. At present, there are two relatively robust techniques to probe the metric of $\mathrm{BH}$ candidates; that is, the study of the disk's thermal spectrum and the analysis of the profile of the $\mathrm{K} \alpha$ iron line. However, these techniques can usually constrain only a certain combination of the spin parameter and of possible deviations from the Kerr solution, because a non-Kerr object with a certain spin can likely mimic a Kerr BH with different spin.

In the present paper, I have reconsidered the interpretation of the three QPOs simultaneously detected in the Xray data of GRO J1655-40 proposed in Ref. [50] to test the Kerr nature of the stellar-mass BH candidate in this source. In the Kerr background, the fundamental frequencies associated to the motion of a test particle depend only on the orbital radius $r$, the $\mathrm{BH}$ mass $M$, and the spin parameter $a$. Since three QPOs are observed at the same time, one can argued that they may be generated at the same orbital radius and thus solve the system of three equations for the three fundamental frequencies to find the three variable, $r$, $M$, and $a$. The measurement of the mass $M$ found with this approach is consistent with the one inferred by studying the orbital motion of the companion star with optical observa- tions found in [51], but with a smaller uncertainty. However, in the literature there is also a different measurement reported in [52] and that would be inconsistent with the mass value inferred in [50]. The relativistic precession model provides also an estimate of the $\mathrm{BH}$ spin with quite high precision, but it turns out to be in disagreement with the value found from the analysis of the disk's thermal spectrum and of the iron line profile.

The relativistic precession interpretation of the QPOs can potentially be a quite powerful tool to test the nature of astrophysical BH candidates. In this case, one has to use the mass $M$ inferred from the optical data as an independent measurement and thus solve the system of three equations for the fundamental frequencies of the spacetime to find the orbital radius $r$, the spin parameter $a$, and constrain possible deviations from the Kerr background through the determination of the deformation parameter under consideration. The data of GRO J1655-40 may be consistent with a Kerr BH, but they also allow for significant deviations from the Kerr solution. With the mass measurement of [51], the disagreement between the results of the relativistic precession interpretation and the measurement obtained with the continuumfitting method persists even relaxing the Kerr BH assumption, and for any choice of the deformation parameter. With the mass measurement of Ref. [52], the relativistic precession model and the continuum-fitting method can be consistent in the Johannsen-Psaltis background with non-vanishing $\epsilon_{3}$ The required deformation is $\epsilon_{3} \sim 7$. It is worth noting that the same value was found in [31] by combining the measurements of the continuum-fitting method and of the power of steady jets.

Acknowledgments This work was supported by the NSFC grant No. 11305038, the Shanghai Municipal Education Commission grant for Innovative Programs No. 14ZZ001, the Thousand Young Talents Program, and Fudan University.

Open Access This article is distributed under the terms of the Creative Commons Attribution 4.0 International License (http://creativecomm ons.org/licenses/by/4.0/), which permits unrestricted use, distribution, and reproduction in any medium, provided you give appropriate credit to the original author(s) and the source, provide a link to the Creative Commons license, and indicate if changes were made.

Funded by $\mathrm{SCOAP}^{3}$.

\section{References}

1. B. Carter, Phys. Rev. Lett. 26, 331 (1971)

2. D.C. Robinson, Phys. Rev. Lett. 34, 905 (1975)

3. P.T. Chrusciel, J.L. Costa, M. Heusler, Living Rev. Relat. 15, 7 (2012). arXiv:1205.6112 [gr-qc]

4. R.H. Price, Phys. Rev. D 5, 2419 (1972)

5. R.H. Price, Phys. Rev. D 5, 2439 (1972)

6. C. Bambi, A.D. Dolgov, A.A. Petrov, JCAP 0909, 013 (2009). arXiv:0806.3440 [astro-ph]

7. R. Narayan, New J. Phys. 7, 199 (2005). arXiv:gr-qc/0506078 
8. R. Narayan, J.E. McClintock, New Astron. Rev. 51, 733 (2008). arXiv:0803.0322 [astro-ph]

9. A.E. Broderick, A. Loeb, R. Narayan, Astrophys. J. 701, 1357 (2009). arXiv:0903.1105 [astro-ph.HE]

10. M.A. Abramowicz, W. Kluzniak, J.-P. Lasota, Astron. Astrophys. 396, L31 (2002). arXiv:astro-ph/0207270

11. C. Bambi, Sci. World J. 2013, 204315 (2013). arXiv:1205.4640 [gr-qc]

12. C. Bambi, Mod. Phys. Lett. A 26, 2453 (2011). arXiv:1109.4256 [gr-qc]

13. C. Bambi, Astron. Rev. 8, 4 (2013). arXiv:1301.0361 [gr-qc]

14. S.N. Zhang, W. Cui, W. Chen, Astrophys. J. 482, L155 (1997). arXiv:astro-ph/9704072

15. L.-X. Li, E.R. Zimmerman, R. Narayan, J.E. McClintock, Astrophys. J. Suppl. 157, 335 (2005). arXiv:astro-ph/0411583

16. J.E. McClintock et al., Class. Quant. Gravity 28, 114009 (2011). arXiv:1101.0811 [astro-ph.HE]

17. A.C. Fabian, M.J. Rees, L. Stella, N.E. White, Mon. Not. R. Astron. Soc. 238, 729 (1989)

18. A.C. Fabian, K. Iwasawa, C.S. Reynolds, A.J. Young, Publ. Astron. Soc. Pac. 112, 1145 (2000). arXiv:astro-ph/0004366

19. C.S. Reynolds, M.A. Nowak, Phys. Rep. 377, 389 (2003). arXiv:astro-ph/0212065

20. C. Bambi, E. Barausse, Astrophys. J. 731, 121 (2011). arXiv:1012.2007 [gr-qc]

21. C. Bambi, Astrophys. J. 761, 174 (2012). arXiv:1210.5679 [gr-qc]

22. C. Bambi, Phys. Rev. D 87, 023007 (2013). arXiv:1211.2513 [grqc]

23. C. Bambi, Phys. Rev. D 87, 084039 (2013). arXiv:1303.0624 [grqc]

24. C. Bambi, D. Malafarina, Phys. Rev. D 88, 064022 (2013). arXiv:1307.2106 [gr-qc]

25. C. Bambi, Phys. Lett. B 730, 59 (2014). arXiv:1401.4640 [gr-qc]

26. T. Johannsen, D. Psaltis, Astrophys. J. 773, 57 (2013). arXiv:1202.6069 [astro-ph.HE]

27. D.F. Torres, Nucl. Phys. B 626, 377 (2002). arXiv:hep-ph/0201154

28. Y. Lu, D.F. Torres, Int. J. Mod. Phys. D 12, 63 (2003). arXiv:astro-ph/0205418

29. C. Bambi, JCAP 1308, 055 (2013). arXiv:1305.5409 [gr-qc]

30. C. Bambi, Phys. Rev. D 85, 043002 (2012). arXiv:1201.1638 [grqc]

31. C. Bambi, Phys. Rev. D 86, 123013 (2012). arXiv:1204.6395 [grqc]

32. C. Bambi, K. Freese, Phys. Rev. D 79, 043002 (2009). arXiv:0812.1328 [astro-ph]

33. C. Bambi, N. Yoshida, Class. Quant. Gravity 27, 205006 (2010). arXiv:1004.3149 [gr-qc]

34. C. Bambi, Phys. Rev. D 87, 107501 (2013). arXiv:1304.5691 [grqc]

35. Z. Li, C. Bambi, JCAP 1401, 041 (2014). arXiv:1309.1606 [gr-qc]
36. L. Stella, M. Vietri, Astrophys. J. 492, L59 (1998). arXiv:astro-ph/9709085

37. L. Stella, M. Vietri, Phys. Rev. Lett. 82, 17 (1999). arXiv:astro-ph/9812124

38. L. Stella, M. Vietri, S. Morsink, Astrophys. J. 524, L63 (1999). arXiv:astro-ph/9907346

39. C.A. Perez, A.S. Silbergleit, R.V. Wagoner, D.E. Lehr, Astrophys. J. 476, 589 (1997). arXiv:astro-ph/9601146

40. A.S. Silbergleit, R.V. Wagoner, M. Ortega-Rodriguez, Astrophys. J. 548, 335 (2001). arXiv:astro-ph/0004114

41. S. Kato, Publ. Astron. Soc. Jpn. 53, 1 (2001)

42. M.A. Abramowicz, W. Kluzniak, Astron. Astrophys. 374, L19 (2001). arXiv:astro-ph/0105077

43. M.A. Abramowicz, V. Karas, W. Kluzniak, W.H. Lee, P. Rebusco, Publ. Astron. Soc. Jpn. 55, 467 (2003)

44. G. Torok, M.A. Abramowicz, W. Kluzniak, Z. Stuchlik, Astron. Astrophys. 436, 1 (2005)

45. L. Rezzolla, S. Yoshida, T.J. Maccarone, O. Zanotti. Mon. Not. R. Astron. Soc. 344, L37 (2003). arXiv:astro-ph/0307487

46. J.D. Schnittman, L. Rezzolla, Astrophys. J. 637, L113 (2006). arXiv:astro-ph/0506702

47. Z. Stuchlik, A. Kotrlova, Gen. Relat. Grav. 41, 1305 (2009). arXiv:0812.5066 [astro-ph]

48. T. Johannsen, D. Psaltis, Astrophys. J. 726, 11 (2011). arXiv: 1010.1000 [astro-ph.HE]

49. C. Bambi, JCAP 1209, 014 (2012). arXiv:1205.6348 [gr-qc]

50. S.E. Motta, T.M. Belloni, L. Stella, T. Muoz-Darias, R. Fender, arXiv: 1309.3652 [astro-ph.HE]

51. M.E. Beer, P. Podsiadlowski, Mon. Not. R. Astron. Soc. 331, 351 (2002). arXiv:astro-ph/0109136

52. R. Shafee et al., Astrophys. J. 636, L113 (2006). arXiv:astro-ph/0508302

53. E. Ayon-Beato, A. Garcia, Phys. Lett. B 493, 149 (2000). arXiv:gr-qc/0009077

54. C. Bambi, L. Modesto, Phys. Lett. B 721, 329 (2013). arXiv:1302.6075 [gr-qc]

55. Z. Li, C. Bambi, Phys. Rev. D 87, 124022 (2013). arXiv:1304.6592 [gr-qc]

56. T. Johannsen, D. Psaltis, Phys. Rev. D 83, 124015 (2011). arXiv:1105.3191 [gr-qc]

57. S. Motta, J. Homan, T. Munoz-Darias, P. Casella, T.M. Belloni, B. Hiemstra, M. Mendez, Mon. Not. R. Astron. Soc. 427, 595 (2012). arXiv:1209.0327 [astro-ph.HE]

58. T.E. Strohmayer, Astrophys. J. 552, L49 (2001). arXiv:astro-ph/0104487

59. J.M. Miller, C.S. Reynolds, A.C. Fabian, G. Miniutti, L.C. Gallo, Astrophys. J. 697, 900 (2009). arXiv:0902.2840 [astro-ph.HE]

60. R.C. Reis, A.C. Fabian, J.M. Miller, Mon. Not. R. Astron. Soc. 402, 836 (2010). arXiv:0911.1151 [astro-ph.HE] 